Ramírez, J., González, G. (2016). Dinámica de la cadena priorizada del sector metalmecánico del departamento de Santander en el período 2010-2015. Revista Lebret, 8. Bucaramanga, Colombia: Universidad Santo Tomás, pp. 175 - 199. ISSN: $2145-5996$

\title{
Dinámica de la cadena priorizada del sector metalmecánico del departamento de Santander en el período 2010-2015*
}

\author{
The dynamics of the prioritized chain of the metal mechanics \\ sector in Santander Department - 2010-2015
}

Julio Ramírez Montañez ${ }^{l}$

Gustavo González Sarmiento ${ }^{2}$

\begin{abstract}
Resumen
El propósito central del presente artículo es presentar los aspectos más importantes de la dinámica de la cadena priorizada del sector metalmecánico del departamento de Santander en los últimos cinco años. La producción de la industria de este sector del departamento se ubica en la quinta posición de la cadena metalmecánica del país. En las ventas internacionales, el departamento de Santander aporta el $1,79 \%$ de las exportaciones de la cadena metalmecánica. Los destinos de las exportaciones estuvieron dirigidos en el período 2010-2015 principalmente a los mercados de los países vecinos como Ecuador y Venezuela.
\end{abstract}

Palabras clave

Sector metalmecánico; cadenas priorizadas; departamento de Santander.

Códigos de clasificación JEL: L61, M20

\begin{abstract}
The central purpose of this article is to present the most relevant aspects of the development of the priority chain of the metal mechanic sector of the Department of Santander in the past five years. Similarly, the department of Santander is located in the fifth position of the industrial production of the metal mechanic chain the country. For its part, the department of Santander brings the 1.79 per cent of the exports of the chain. The destination of exports of Santander department of products from the metal mechanic chain were directed in the period 2010-2015 mainly to markets in neighboring like Ecuador and Venezuela.
\end{abstract}

\section{Keywords}

The metal mechanic sector; prioritized chains; department of Santander.

* Artículo derivado del proyecto de investigación "Dinámica relativa del comercio intrarregional de los sectores metalmecánico y autopartes con los países de la Alianza del Pacífico" Grupo GRICANI Facultad de Administración de Negocios Internacionales, Universidad Pontificia Bolivariana, Bucaramanga - Grupo por la Integración y la Globalización de los Negocios. Facultad de Negocios Universidad Santo Tomás Seccional Bucaramanga.

1 Magíster en Relaciones Internacionales, docente Facultad de Administración de Negocios Internacionales Universidad Pontificia Bolivariana Seccional Bucaramanga. Correo electrónico: julio.ramirez@upb.edu.co

2 Especialista en Desarrollo Regional, docente Facultad de Negocios Universidad Santo Tomás, Bucaramanga. Correo electrónico: gusta_gsar@hotmail.com 


\section{Introducción}

Para una economía inserta en el mundo globalizado, es cada vez más importante identificar los sectores donde existe un potencial para el aumento de la productividad, con el ánimo de poder crear los entornos adecuados y los incentivos correctos que impulsen los aumentos de productividad. En este sentido, las medidas encaminadas a procurar estos entornos para el desarrollo productivo normalmente se han enfocado al ámbito macroeconómico.

El Ministerio de Comercio, Industria y Turismo de Colombia creó el Programa de Transformación Productiva (PTP) en el 2008, con el fin de establecer la Política Industrial de Desarrollo Productivo del país, con la que se buscaba generar entornos más competitivos y empresas más fuertes y productivas. El PTP desde su creación ha sido un aliado de los empresarios colombianos al ofrecer acompañamiento para acelerar su productividad y competitividad, orientando sus capacidades y aprovechando sus ventajas comparativas.

Las metas del Gobierno de Colombia con el Programa de Transformación Productiva están orientadas a incrementar las exportaciones de bienes no minero energéticos y de servicios en los próximos años a US\$30.000 millones, captar US\$ 6000 millones de divisas por concepto del turismo y lograr que más de 4100 empresas se conviertan en exportadoras. (PTP, 2016). Este programa se ha convertido en un promotor de la productividad y competitividad de las empresas colombianas a través de cinco frentes: fomentar mejoras en la productividad y la calidad, optimizar las reglas de juego, potenciar procesos para ser más competitivos, generar capacidades y facilitar el acceso a la financiación (PTP, 2016).

Sin embargo, el logro más importante de este programa gubernamental ha sido seleccionar veinte sectores de gran potencialidad de convertirse en industrias de clase mundial. Los sectores vinculados al PTP cuentan con planes de negocio diseñados a la medida que definen el curso por seguir para alcanzar incrementos decisivos en la productividad y competitividad, mientras fortalecen sus cadenas productivas y se posicionan en mercados internacionales. Estos sectores identificados en Colombia y que el Programa de Transformación Productiva define como priorizados en búsqueda de una diversificación de la oferta exportable son:

Tabla 1. Sectores priorizados en Colombia

\begin{tabular}{ll}
\hline \multicolumn{1}{c}{ Sector } & \multicolumn{1}{c}{ Sectores priorizados en Colombia } \\
\hline Sectores de servicios & $\begin{array}{l}\text { BPO\&O, Software y TI, Energía Eléctrica, Bienes y Servicios Conexos, Turismo de } \\
\text { Salud, Turismo de Bienestar, Turismo de Naturaleza. }\end{array}$ \\
\hline \multirow{2}{*}{ Sectores de manufacturas } & $\begin{array}{l}\text { Cosméticos y Aseo, Editorial e Industria de la Comunicación Gráfica, Autopartes y } \\
\text { Vehículos, Textil y Confecciones (Cuero, Calzado y Marroquinería), Metalmecánico, } \\
\text { Siderúrgico, Astillero. }\end{array}$ \\
\hline \multirow{2}{*}{ Sectores de agroindustria } & $\begin{array}{l}\text { Acuícola, Carne Bovina, Lácteo, Palma, Aceites, Grasas Vegetales y Biocombustibles, } \\
\text { Chocolatería, Confitería y Materias Primas, Hortofrutícola. }\end{array}$ \\
\hline
\end{tabular}

Fuente: Programa de Transformación Productiva, 2016. Elaboración propia. 
Estos sectores priorizados por el PTP representan el 34\% de las exportaciones no minero energéticas de Colombia. La Política Industrial está enfocada al apoyo y promoción de estas industrias y el PTP es un vehículo de ejecución focalizado en sectores con potencial de crecimiento en ventas y exportaciones (PTP, 2016).

Desde el segundo semestre del 2016, representantes del Programa de Transformación Productiva PTP y del Ministerio de Comercio, Industria y Turismo han venido trabajando en la implementación del Programa Colombia Moderna - Colombia Productiva con una serie de actores regionales del departamento de Santander.

En el proceso de implementación de este programa, el Gobierno, la empresa y la academia se han articulado de la mano de representantes de la Oficina ProColombia Santander, la Comisión Regional de Competitividad de Santander, la Cámara de Comercio de Bucaramanga, la Cámara de Comercio de Barrancabermeja, los gremios de la producción y los grupos de investigación de facultades de negocios internacionales del Área Metropolitana de Bucaramanga.

Este programa denominado Colombia Moderna - Colombia Productiva se basa en la priorización de cadenas productivas. En todo el país el Programa de Transformación Productiva ha detectado 1047 priorizaciones sectoriales y sus objetivos principales están dirigidos a mejorar las condiciones del entorno competitivo de las empresas mediante la coordinación entre las entidades y actores que tengan que ver con el comercio exterior de las regiones y el desarrollo de instrumentos específicos para atender sus necesidades.

La metodología para la implementación del Programa Colombia Moderna Colombia Productiva en el departamento de Santander se ha desarrollado utilizando la denominada "Metodología de Priorización" la cual se encuentra distribuida en 3 fases:

Fase 1. Creación de equipos de trabajo en los departamentos.

Fase 2. Creación de bases de datos para reflejar el sistema productivo del país.

Fase 3. Priorización con enfoques de potencialidad de exportaciones.

De acuerdo con el análisis realizado en el desarrollo de las tres fases, se han detectado seis cadenas productivas con potencial exportador en el departamento de Santander. Estas cadenas son: químicos, metalmecánico, industrias 4.0, moda, agroalimentario y turismo.

En el desarrollo de la fase 3, luego de debatir las diversas propuestas sobre cuáles deberían ser los subsectores indicados para desarrollar proyectos que lleven a un aumento en las exportaciones del departamento de Santander y utilizando algunos factores de medición como el aporte al Producto Interno Bruto regional, el número de empresas del subsector y el desarrollo de un clúster, se llegó a la decisión de elegir los siguientes subsectores: 
Tabla 2. Sectores priorizados de Santander

\begin{tabular}{ll}
\multicolumn{1}{c}{ Sector } & \multicolumn{1}{c}{ Subsector } \\
\hline Metalmecánico & Productos elaborados de metal \\
\hline Moda & Confecciones \\
\hline Agroalimentario & Frutas bebestibles \\
\hline Químico & Dispositivos médicos \\
\hline Turismo & Corredor turístico de Santander \\
\hline Industrias 4.0 & BPO, KPO. \\
\hline
\end{tabular}

Fuente: Programa de Transformación Productiva, 2016. Elaboración propia.

En la tercera sesión de las Jornadas de Transformación Productiva se tomó la decisión de apoyar los subsectores anteriormente mencionados y específicamente para el sector metalmecánico se tomó la decisión de continuar el proceso con los productos elaborados de metal. Los capítulos arancelarios con potencial exportador del departamento de Santander de la cadena priorizada del sector metalmecánico son los comprendidos entre los capítulos arancelarios 82 y 85 .

Tabla 3. Capítulos arancelarios de la cadena priorizada del sector metalmecánico de Santander

\begin{tabular}{ll}
\hline Capítulos arancelarios & Descripción capítulo arancelario \\
\hline Capítulo 82 & $\begin{array}{l}\text { Herramientas y útiles, artículos de } \\
\text { cuchillería y cubiertos de mesa de metal } \\
\text { común, partes de estos. }\end{array}$ \\
\hline Capítulo 83 & Manufacturas diversas de metal común. \\
\hline Capítulo 84 & $\begin{array}{l}\text { Reactores nucleares, calderas, máquinas, } \\
\text { aparatos y artefactos mecánicos; partes } \\
\text { de estas máquinas o aparatos. }\end{array}$ \\
\hline Capítulo 85 & $\begin{array}{l}\text { Máquinas, aparatos y material eléctrico } \\
\text { y sus partes; aparatos de grabación o } \\
\text { reproducción de sonido, aparatos de } \\
\text { grabación o reproducción de imagen } \\
\text { y sonido en televisión y las partes y } \\
\text { accesorios de estos aparatos. }\end{array}$ \\
\hline
\end{tabular}

Fuente: SIEX-DIAN 2016. Elaboración propia.

\section{Fundamentación teórica}

Los nuevos paradigmas que marcan la vanguardia en el comercio internacional hablan de la necesidad de insertarse en las cadenas globales de valor antes de continuar con un modelo de intercambio basado en países y bloques (Kosakoff $\&$ López, 2008) 
Los industriales piensan hoy en día en cómo lograr encadenamientos productivos que permitan que sus bienes se integren en procesos internacionales sacando ventaja de los acuerdos de libre comercio. Siendo estos encadenamientos la llave para ingresar con vigor a las cadenas globales de valor, lo que se traduce en una mayor fortaleza y competividad para las industrias de los países (Dalle, Fossati \& Lavopal, 2013).

Los encadenamientos productivos, la competitividad, la productividad basada en la innovación y la internacionalización de las empresas, son los cuatro ejes principales de la política industrial moderna de un país para que los empresarios crezcan, compitan, generen empleo y conquisten nuevos mercados (Humphrey \& Schimtz, 2002).

De acuerdo con Tomta \& Chiatchoua (2009) el concepto de cadenas productivas se refiere, en su sentido más estricto, a todas las etapas comprendidas en la elaboración, distribución y comercialización de un bien o servicio hasta su consumo final. En otras palabras, se puede analizar una cadena productiva desde una perspectiva de los factores de producción. Es un conjunto de agentes económicos que participan directamente en la producción, transformación y el traslado hacia el mercado de un mismo producto. Tiene como principal objetivo localizar las empresas, las instituciones, las operaciones, las dimensiones y capacidades de negociación, las tecnologías, las relaciones de producción y las relaciones de poder en la determinación de los precios.

López (2003) por su parte, define una cadena productiva como un sistema conformado por la interacción en armonía entre diversos participantes, directa o indirectamente, en la producción y consumo de productos y servicios. Mientras que Lima et al. (2001) mencionan que las cadenas productivas son conjuntos de actores sociales, como sistemas productivos, proveedores de servicios e insumos, industrias de procesamiento y transformación, distribución y comercialización, además de consumidores finales del producto y subproductos.

De igual forma, Isaza (2008) señala que este es un concepto que proviene de la escuela de la planeación estratégica. Según esta escuela, la competitividad de una empresa se explica no solo a partir de sus características internas a nivel organizacional, sino que también está determinada por factores externos asociados a su entorno. En tal sentido, las relaciones con proveedores, el Estado, los clientes y los distribuidores, entre otros, generan estímulos y permiten sinergias que facilitan la creación de ventajas competitivas. Así, la cadena productiva puede definirse como un conjunto estructurado de procesos de producción que tiene en común un mismo mercado y en el que las características tecno productivas de cada eslabón afectan la eficiencia y productividad de la producción en su conjunto (Solvell, 2008). 


\section{Cadenas productivas: Descripción y evolución}

El análisis del desarrollo económico a través del esquema de cadenas productivas es una metodología que se ha venido desarrollando en el plano internacional desde la primera mitad del siglo XX. En el ámbito internacional los primeros acercamientos al concepto de cadena productiva parten de las teorías de eslabonamientos propuestas por Albert Hirschman en 1958, definidas como el conjunto de fuerzas que generan inversiones y que son accionadas cuando la capacidad productiva de los sectores que producen insumos para esa línea y que utilizan los productos de esta es insuficiente (Castellanos \& Rojas, 2001)

Posteriormente, Richardson en 1972 profundiza en la perspectiva de interacción, cuando sostiene que la subcontratación se ha vuelto más difundida, y afirma que un distribuidor grande - más allá de ser una cadena de distribución tiene la capacidad de diseñar y construir patrones complejos de actividades coordinadas entre las empresas, enfatizando que esta capacidad de coordinar surge sin ningún tipo de participación accionaria entre los proveedores (Richardson, 1972).

A continuación, en 1983, se desarrolla un concepto que identifica un nuevo mecanismo de interacción empresarial, relacionado con la teoría de eslabonamientos propuesta por Hirschman, y que define los "Distritos Industriales", como 'conjuntos de unidades productivas especializadas en un determinado sector y concentradas en un territorio restringido, geográfica e históricamente (Stumpo, 1996).

Estos distritos industriales se caracterizan por la existencia de fuertes asociaciones sectoriales. Los distritos industriales se distinguen, entre otras características, por contar con una colaboración interempresarial cercana, una identidad sociocultural que facilita la confianza mutua y una interacción de variables sociales, económicas y culturales que caracterizan su estructura productiva (Bagnasco, 2000).

Sin embargo, el principal aporte conceptual de las cadenas productivas es realizado por Michael Porter en su libro La ventaja competitiva de las naciones en 1990 planteó el concepto de "cadena de valor" para describir el conjunto de actividades que se llevan a cabo al competir en un sector y que se pueden agrupar en dos categorías: en primer lugar están aquellas relacionadas con la producción, comercialización, entrega y servicio de posventa; en segundo lugar se ubicarían las actividades que proporcionan recursos humanos y tecnológicos, insumos e infraestructura (Porter, 1990).

Porter (1990) en cuanto a los encadenamientos productivos señala que la cadena de valor de una empresa es un sistema interdependiente o red de actividades, conectado mediante enlaces. Los enlaces se producen cuando la forma de llevar a cabo una actividad afecta el coste o la eficacia de otras actividades. Frecuentemente, los enlaces crean situaciones en las que si se opta por algo tiene que ser a cambio de renunciar a otra cosa, sobre todo en lo que se refiere a la realización de diferentes actividades que deban optimizarse. 
En los últimos años ha surgido otro enfoque de análisis del concepto de cadenas productivas, el cual ha estado encabezado por la Organización del Desarrollo Industria de la Organización de Naciones Unidas (ONUDI) donde se toma como punto de referencia la llamada competitividad sistémica. Para este enfoque, el Estado ya no está en el centro del proceso de configuración de políticas de desarrollo productivo en el ámbito local, regional o nacional, sino que, en su reemplazo, se sitúan junto a este toda una red de actores sociales: las firmas, las instituciones de apoyo, las agremiaciones de empresarios y los gobiernos locales. En tal sentido, el marco de análisis para el diseño de políticas de desarrollo productivo se enriquece con el concepto de clúster. De acuerdo con la Organización del Desarrollo Industria de la Organización de Naciones Unidas, los clúster son aglomeraciones geográficas de empresas que producen y venden productos relacionados o complementarios, por lo cual tienen retos y oportunidades comunes (ONUDI, 2005).

\section{De la cadena productiva al clúster}

En la actualidad, el desarrollo regional y la teoría que le da consistencia lógica regresaron hacia uno de los principales teóricos de los inicios del siglo XX: Alfred Marshall. Este economista identificó tres distintas razones para la localización industrial: primera, para concentrar un cierto número de firmas en un mismo lugar, un centro industrial hace posible adquirir e intercambiar trabajadores especializados, lo que beneficia a todas las empresas que los requieran; segunda, un centro industrial permite que la industria se provea de insumos específicos no comercializados, lo que se efectúa en gran variedad y a bajo costo; y tercera, porque localmente la información fluye con mayor facilidad que a grandes distancias, un centro industrial genera lo que nosotros podríamos ahora llamar transferencia tecnológica (Corrales y Salvador, 2007).

Corrales y Salvador (2007) argumentan que al generalizarse la globalización de las economías, las regiones experimentaron cambios profundos para adecuarse a los avances tecnológicos y a las nuevas estrategias de segmentar los procesos de producción que han modificado la competitividad de las empresas. Estas transformaciones condujeron a nuevas formas de organización económica y empresarial para enfrentar la competencia, lo cual condujo a la formación del clúster, que simboliza el desarrollo regional en los tiempos modernos.

De acuerdo con McCormick (2005) los clúster son concentraciones geográficas de empresas interconectadas, suministradores especializados, proveedores de servicios, de universidades, institutos de normalización, asociaciones comerciales que compiten pero que también cooperan. Por su parte, Barrientos (2011) define el concepto de clúster como un grupo geográficamente denso de empresas e instituciones conexas, pertenecientes a un campo concreto, unidas por rasgos comunes y complementarios entre sí. Por su dimensión geográfica, un clúster puede ser urbano, regional, nacional o incluso supranacional. 
Barrientos (2011) señala que los clúster adoptan varias formas, dependiendo de su profundidad y complejidad, pero la mayoría de ellos comprenden empresas de productos o servicios finales, proveedores de materiales, componentes, maquinaria y servicios especializados, instituciones financieras y empresas de sectores afines. De igual forma, Lazzeretti (2006) establece que en dichos clústeres también suelen integrarse empresas que constituyen eslabones posteriores de la cadena, es decir, canales de distribución o cliente; fabricantes de productos complementarios; proveedores de infraestructura; las instituciones públicas y privadas que facilitan formación, información, investigación y apoyo técnico especializado como universidades, grupos de reflexión, entidades de formación profesional y los institutos de normalización. Los organismos del Estado que influyen significativamente en un clúster pueden considerarse parte de él.

\section{Metodología}

Para el desarrollo de la investigación se aplicó un análisis documental a través de fuentes secundarias de información en los últimos cinco años, tomando como referencia las variables de empleo, valor agregado, producción bruta, distribución regional de la producción y principales productos exportados y destinos de las exportaciones de la cadena priorizada del sector metalmecánico de Santander, utilizando las fuentes como Departamento Administrativo Nacional de Estadística (DANE), del Sistema Integrado de Estadísticas de Comercio Exterior SIEX y del Programa de Transformación Productiva del Ministerio de Comercio Industria y Turismo de Colombia.

\section{Resultados}

En esta sección se presenta el análisis del sector metalmecánico del departamento de Santander en los últimos cinco años, tomando como referencia las variables de empleo, valor agregado, producción bruta, distribución regional de la producción y principales productos exportados y destinos de las exportaciones.

\section{Empleo de la cadena del sector metalmecánico}

Para realizar el análisis del empleo en la cadena productiva del sector metalmecánico de Colombia se han usado las variables de distribución regional del empleo en los departamentos colombianos, la evolución del empleo en Colombia y Santander y el comportamiento del empleo en las principales áreas metropolitanas de Colombia.

En el 2015 el sector aportó la generación de 203.386 puestos de trabajo en la economía colombiana, de los cuales 9502 se generaron en el departamento de Santander y 6066 en el Área Metropolitana de Bucaramanga. 
En la distribución regional del empleo de la cadena productiva del sector metalmecánico de Colombia, los departamentos que concentran el mayor número de empleos en la cadena son Cundinamarca con el 30\% de contribución al empleo de la cadena del sector metalmecánico, seguido por el departamento de Antioquia con $16.6 \%$ y en tercer lugar el departamento del Valle del Cauca con $12.6 \%$.

El departamento de Santander se ubica en el quinto puesto concentrando el 4,7\% del empleo de la cadena metalmecánica a nivel nacional.

Tabla 4. Distribución regional del empleo

\begin{tabular}{lc}
\hline \multicolumn{1}{c}{ Departamento } & \% de Empleo \\
\hline Antioquia & $16,6 \%$ \\
\hline Atlántico & $6,9 \%$ \\
\hline Bolívar & $3,3 \%$ \\
\hline Boyacá & $3,4 \%$ \\
\hline Caldas & $2,6 \%$ \\
\hline Caquetá & $0,7 \%$ \\
\hline Cauca & $1,9 \%$ \\
\hline Cesar & $1,8 \%$ \\
\hline Chocó & $0,4 \%$ \\
\hline Córdoba & $0,9 \%$ \\
\hline Cundinamarca & $30 \%$ \\
\hline Huila & $1,1 \%$ \\
\hline La Guajira & $0,8 \%$ \\
\hline Magdalena & $1,4 \%$ \\
\hline Meta & $1,5 \%$ \\
\hline Nariño & $1,5 \%$ \\
\hline Norte de Santander & $2,3 \%$ \\
\hline Quindío & $0,7 \%$ \\
\hline Santander & $4,7 \%$ \\
\hline Sucre & $0,8 \%$ \\
\hline Tolima & $1,6 \%$ \\
\hline Valle & $12,6 \%$ \\
\hline Fuente: DANE & \\
\hline Cor & \\
\hline
\end{tabular}

Fuente: DANE. (2016). Elaboración propia.

En cuanto al comportamiento del empleo de la cadena del sector metalmecánico se puede apreciar en la gráfica presentada a continuación un crecimiento continuo 
del empleo en Colombia. Este sector tiene una evolución en esta variable de análisis al pasar de emplear 160.529 personas en el 2010 a 203.386 en el 2015, alcanzando un crecimiento anual promedio de $4.8 \%$ en el período $2010-2015$.

En cuanto al comportamiento del empleo de la cadena del sector metalmecánico se puede apreciar en la gráfica presentada a continuación un crecimiento continuo del empleo en Colombia, alcanzando un crecimiento anual promedio de $4.8 \%$. En cuanto al departamento de Santander la cantidad de puestos de trabajo en el período 20102015 oscila entre seis mil y diez mil. Se destaca una fuerte caída en el 2011, derivada del deterioro de las relaciones en este año con los países vecinos de Colombia y Ecuador. Posteriormente desde el 2012 tiene una recuperación considerable al aumentar 3830 puestos de trabajo en el 2013, hasta alcanzar un crecimiento anual promedio en los últimos cinco años de $0.9 \%$.

Figura 1. Evolución del empleo de la cadena metalmecánica

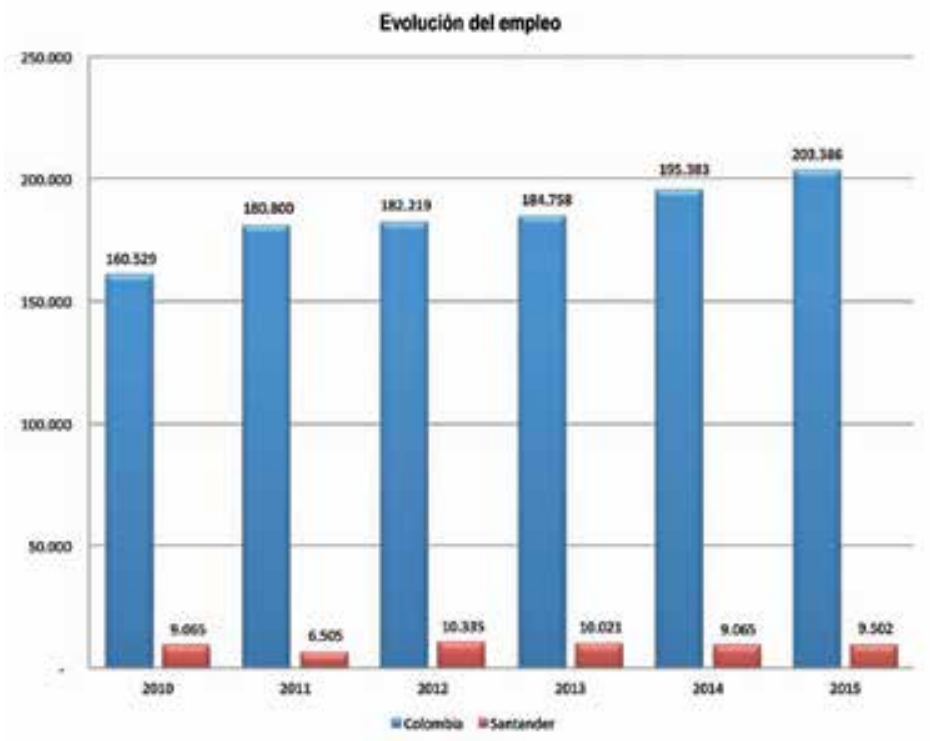

Fuente: DANE (2016). Elaboración propia.

El indicador de empleo de la cadena del sector metalmecánico en las principales áreas metropolitanas de Colombia tuvo un desempeño positivo al tener un crecimiento de $2.4 \%$ durante los últimos cinco años. Bogotá es el área metropolitana con mayor número de puestos de trabajo en el país al generar 47.274 en el 2015. El área metropolitana con mejor desempeño fue Pereira, realizando un aporte significativo a la generación de empleo en la cadena del sector metalmecánico con un $14.6 \%$ del total de puestos de trabajo del sector en Colombia, seguido por Cartagena con un $10.7 \%$ y en tercer lugar Villavicencio con un $8.4 \%$. 
Es de desatacar que en todas las áreas metropolitanas de Colombia se presentó un crecimiento positivo del empleo de la cadena metalmecánica a excepción de Cúcuta $-1.4 \%$, Montería $-1.7 \%$ y Bucaramanga con $-3.3 \%$. Se resalta de igual forma, la importante caída que ha sufrido la cadena metalmecánica del Área Metropolitana de Bucaramanga al ser el área con una mayor caída en la generación de empleo del sector en el período 2010-2015 al decrecer en un 3.3\% (DANE, 2016).

Tabla 5. Empleo según área metropolitana

\begin{tabular}{lccccccc}
\hline $\begin{array}{c}\text { Área } \\
\text { metropolitana }\end{array}$ & $\mathbf{2 0 1 0}$ & $\mathbf{2 0 1 1}$ & $\mathbf{2 0 1 2}$ & $\mathbf{2 0 1 3}$ & $\mathbf{2 0 1 4}$ & $\mathbf{2 0 1 5}$ & $\begin{array}{c}\text { Crecimiento } \\
\text { promedio }\end{array}$ \\
\hline Barranquilla & 8.305 & 10.339 & 9.396 & 9.217 & 9.887 & 11.370 & $6.5 \%$ \\
\hline Bogotá & 46.962 & 52.877 & 41.242 & 50.460 & 50.038 & 47.274 & $0.1 \%$ \\
\hline Bucaramanga & 7.174 & 5.289 & 6.459 & 6.494 & 7.263 & 6.066 & $-3.3 \%$ \\
\hline Cali & 14.478 & 15.476 & 16.826 & 15.368 & 19.716 & 17.119 & $3.4 \%$ \\
\hline Cartagena & 2.844 & 3.677 & 3.060 & 4.588 & 4.279 & 4.737 & $10.7 \%$ \\
\hline Cúcuta & 3.537 & 3.291 & 3.844 & 3.392 & 4.265 & 3.304 & $-1.4 \%$ \\
\hline Ibagué & 1.542 & 2.117 & 2.107 & 2.607 & 2.395 & 2.136 & $6.7 \%$ \\
\hline Manizales & 2.999 & 3.334 & 3.056 & 3.756 & 3.969 & 4.092 & $6.4 \%$ \\
\hline Medellín & 19.328 & 20.904 & 22.467 & 21.816 & 23.005 & 22.605 & $3.2 \%$ \\
\hline Montería & 1.760 & 1.945 & 1.821 & 2.200 & 2.122 & 2.639 & $8.4 \%$ \\
\hline Pasto & $\mathbf{1 1 2 . 7 7 1}$ & $\mathbf{1 2 3 . 6 7 9}$ & $\mathbf{1 1 4 . 9 2 1}$ & $\mathbf{1 2 4 . 7 1 1}$ & $\mathbf{1 3 2 . 3 1 3}$ & $\mathbf{1 2 3 . 8 8 3}$ & $\mathbf{2 . 4 \%}$ \\
\hline Pereira & 755 & 885 & 748 & 794 & 859 & $-1.7 \%$ \\
\hline Villavicencio & 1.239 & 1.321 & 1.762 & 1.806 & 1.400 & $2.3 \%$ \\
\hline Total áreas & 1.659 & 2.436 & 2.437 & 2.304 & 2.773 & 3.283 & $14.6 \%$ \\
\hline & & & & & & & \\
\hline
\end{tabular}

Fuente: DANE (2016). Elaboración propia.

\section{Evolución de la producción bruta del sector metalmecánico}

En esta variable es de destacar el muy buen comportamiento de la producción de la cadena del sector metalmecánico de Colombia, al tener un crecimiento anual promedio de $3.3 \%$ y sobre todo el excelente comportamiento del departamento de Santander al alcanzar un crecimiento del 13\%, al pasar de \$131 mil millones en el 2010 hasta \$214 mil millones en el 2014, siendo este el mejor año de producción. 
Figura 2. Evolución de la producción bruta de la cadena
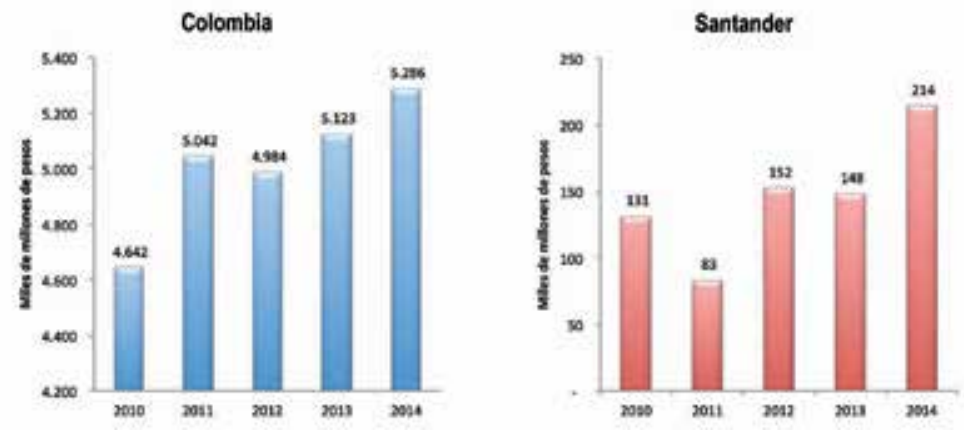

Fuente: DANE (2016). Elaboración propia.

El mejoramiento en el valor agregado de la producción industrial de la cadena metalmecánica ha sido uno de los objetivos primordiales del Programa de Transformación Productiva para el sector metalmecánico de Colombia y Santander. El valor agregado de la cadena metalmecánica en Colombia tuvo un crecimiento del $3.7 \%$ pasando de vender \$1935 millones en el 2010 a vender \$2238 millones en el 2015. Por su parte, el departamento de Santander tuvo un considerable crecimiento anual promedio de $9.2 \%$ en el período $2010-2015$, pasando de vender 60 mil millones de pesos en 2010 a 85 mil millones en el 2015.

Figura 3. Evolución del valor agregado del sector metalmecánico en Colombia y el departamento de Santander
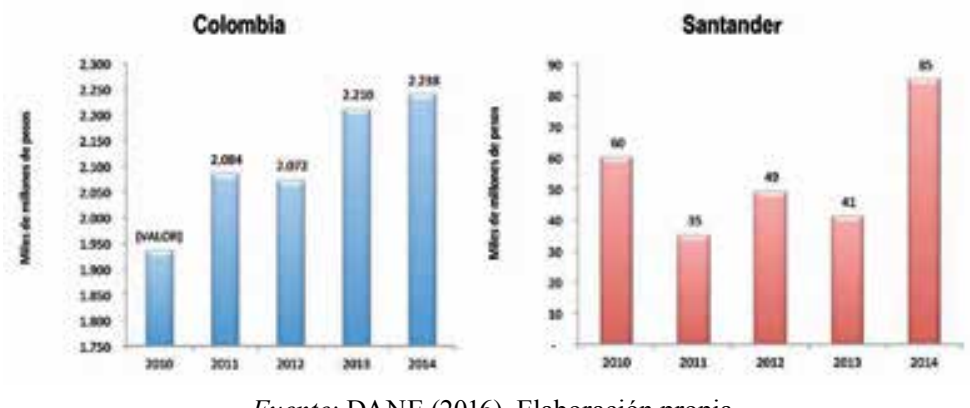

Fuente: DANE (2016). Elaboración propia.

El departamento de Antioquia es el que tiene una mayor producción del sector metalmecánico de Colombia, con una participación del 24\%, seguido por el departamento de Cundinamarca con $17.1 \%$ y en tercer lugar el departamento del Atlántico con el 7.7\%. El departamento de Santander se ubica en la quinta posición con $4.1 \%$ de la producción de la cadena metalmecánica del país. 
Tabla 6. Distribución de la producción del sector por departamento

\begin{tabular}{lc}
\hline \multicolumn{1}{c}{ Departamento } & \% de producción \\
\hline Antioquia & $24,0 \%$ \\
\hline Atlántico & $7,7 \%$ \\
\hline Bolívar & $1,3 \%$ \\
\hline Boyacá & $0,8 \%$ \\
\hline Caldas & $3,6 \%$ \\
\hline Cundinamarca & $17,1 \%$ \\
\hline Norte de Santander & $0,6 \%$ \\
\hline Risaralda & $0,5 \%$ \\
\hline Santander & $4,1 \%$ \\
\hline Tolima & $0,2 \%$ \\
\hline Valle & $6,3 \%$ \\
\hline
\end{tabular}

Fuente: DANE (2016). Elaboración propia.

En Colombia se encuentran registradas en las diferentes Cámaras de Comercio un total de 4090 empresas de la cadena metalmecánica. La mayor concentración de empresas del sector metalmecánico se encuentra en los departamentos de Antioquia con 549, representado un 13,4\%, seguida en segundo lugar por el departamento del Valle del Cauca con 449 empresas, que representan el 11\% y, el departamento del Atlántico con 413 empresas, con un 10,1\%. Por su parte el departamento de Santander concentra 130 empresas en su área geográfica equivalentes al 3,2\% de las empresas de la cadena.

Tabla 7. Distribución regional de las empresas

\begin{tabular}{lc}
\hline \multicolumn{1}{c}{ Departamento } & \% de concentración de la cadena \\
\hline Antioquia & $13,4 \%$ \\
\hline Atlántico & $10,1 \%$ \\
\hline Bolívar & $1,9 \%$ \\
\hline Boyacá & $1,4 \%$ \\
\hline Caldas & $1,0 \%$ \\
\hline Casanare & $1,1 \%$ \\
\hline Cauca & $0,2 \%$ \\
\hline Cesar & $0,7 \%$ \\
\hline Córdoba & $0,4 \%$ \\
\hline Cundinamarca & $6,4 \%$ \\
\hline Huila & $0,5 \%$ \\
\hline
\end{tabular}




\begin{tabular}{ll}
\hline Magdalena & $0,6 \%$ \\
\hline Meta & $0,9 \%$ \\
\hline Nariño & $0,5 \%$ \\
\hline Norte de Santander & $0,5 \%$ \\
\hline Putumayo & $0,2 \%$ \\
\hline Quindío & $0,5 \%$ \\
\hline Santander & $3,2 \%$ \\
\hline Sucre & $0,1 \%$ \\
\hline Tolima & $0,8 \%$ \\
\hline Valle & $11,0 \%$ \\
\hline
\end{tabular}

Fuente: DANE (2016). Elaboración propia.

En la distribución de las 4090 empresas del sector metalmecánico en Colombia se ha determinado clasificarlas en mipymes y grandes empresas. El 98.2\% de las empresas del sector metalmecánico en Colombia son mipymes con un total de 4013, de las cuales 127 están ubicadas en el departamento de Santander. El departamento que cuenta con la mayor parte de estas empresas es Cundinamarca con 1988, siendo el departamento que más aporta en esta variable. Otros departamentos destacados son Antioquia con 539 mypimes, Atlántico con 410 y el Valle con 439.

Tabla 8. Empresas mipymes del sector metalmecánico en Colombia

\begin{tabular}{lc}
\hline \multicolumn{1}{c}{ Departamento } & Número de empresas \\
\hline Amazonas & 1 \\
\hline Antioquia & 539 \\
\hline Arauca & 2 \\
\hline Atlántico & 410 \\
\hline Bolívar & 75 \\
\hline Boyacá & 56 \\
\hline Caldas & 38 \\
\hline Caquetá & 2 \\
\hline Casanare & 47 \\
\hline Cauca & 5 \\
\hline Cesar & 29 \\
\hline Chocó & 2 \\
\hline Córdoba & 17 \\
\hline Cundinamarca & 1988 \\
\hline Huila & 20 \\
\hline & \\
\hline
\end{tabular}


Dinámica de la cadena priorizada del sector metalmecánico del departamento de Santander...

\begin{tabular}{lc}
\hline Magdalena & 23 \\
\hline Meta & 37 \\
\hline Nariño & 21 \\
\hline Norte de Santander & 21 \\
\hline Putumayo & 10 \\
\hline Quindío & 22 \\
\hline Risaralda & 45 \\
\hline Santander & 127 \\
\hline Sucre & 5 \\
\hline Tolima & 32 \\
\hline Valle & 439 \\
\hline Total & $\mathbf{4 0 1 3}$ \\
\hline
\end{tabular}

Fuente: DATLAS (2016). Elaboración propia.

La tabla 9 refleja la distribución de las 77 empresas grandes de la cadena metalmecánica de Colombia. El departamento de Cundinamarca es el precursor de este tipo de empresas al contar con 33 en su territorio, aportando el $42 \%$ del total nacional. Los departamentos del Valle y Antioquia aportan cada una 10 grandes empresas al total nacional. El departamento de Santander se encuentra en el quinto lugar con 3 empresas consideradas grandes. Ellas son Industrias AVM, Metalteco y Transejes.

Tabla 9. Empresas grandes del sector metalmecánico en Colombia

\begin{tabular}{lc}
\multicolumn{1}{c}{ Departamento } & Número de empresas \\
\hline Antioquia & 10 \\
\hline Atlántico & 3 \\
\hline Bolívar & 3 \\
\hline Boyacá & 1 \\
\hline Caldas & 4 \\
\hline Cauca & 3 \\
\hline Cundinamarca & 33 \\
\hline Risaralda & 1 \\
\hline Santander & 3 \\
\hline Valle & 10 \\
\hline Total & $\mathbf{7 7}$ \\
\hline
\end{tabular}

Fuente. DANE (2016). Elaboración propia. 
El departamento de Santander cuenta con 130 empresas del sector metalmecánico inscritas en las diversas cámaras de comercio del departamento. El cuadro 10 muestra que el Área Metropolitana de Bucaramanga compuesta por los municipios de Bucaramanga, Floridablanca, Piedecuesta y Girón es la zona geográfica que tiene mayor densidad de empresas del sector metalmecánico al sumar un total de 89 empresas. La ciudad de Bucaramanga es la que se destaca con un mayor número de empresas de la cadena del sector metalmecánico con 68 empresas correspondientes al 52\% del total de empresas del departamento de Santander.

La ciudad de Barrancabermeja, con toda la experiencia del sector metalmecánico al servicio de la industria petrolera, cuenta con 32 empresas que se dedican principalmente al mantenimiento y reparación de maquinaria y equipo y a la fabricación de productos metalmecánicos. Es de destacarse el caso de la población de Sabana de Torres que aporta dos empresas a la cadena del sector metalmecánico del departamento de Santander.

Tabla 10. Empresas del sector metalmecánico en Santander

\begin{tabular}{lcc}
\hline \multicolumn{1}{c}{ Localidad } & Número de empresas & Porcentaje \\
\hline Bucaramanga & 68 empresas & $52 \%$ \\
\hline Barrancabermeja & 32 Empresas & $25 \%$ \\
\hline Floridablanca & 11 Empresas & $9 \%$ \\
\hline Girón & 5 Empresas & $5 \%$ \\
\hline Piedecuesta & 5 Empresas & $5 \%$ \\
\hline Málaga & 2 Empresas & $2 \%$ \\
\hline Sabana De Torres & 2 Empresas & $2 \%$ \\
\hline Total & $\mathbf{1 3 0}$ Empresas & $\mathbf{1 0 0} \%$ \\
\hline
\end{tabular}

Fuente: Compite 360. Elaboración propia.

En la tabla 11 se presentan los aspectos más relevantes de las principales empresas del Área Metropolitana de Bucaramanga, teniendo en cuenta las características de la empresa y los productos ofrecidos. La mayoría de las empresas ubicadas en el Área Metropolitana de Bucaramanga que hacen parte de la cadena metalmecánica enfocada su producción principalmente en la fabricación de maquinaria industrial para el sector agroalimentario, productos materiales para la construcción y productos de autopartes, tal y como se evidencia en la tabla presentada a continuación. 
Tabla 11. Caracterización de las empresas metalmecánicas A.M.B

\begin{tabular}{|c|c|c|}
\hline Empresa & Principales productos ofrecidos & Características de la empresa \\
\hline INDUSTRIAS A.V.M & $\begin{array}{l}\text { Productos para el sector agroindustrial: } \\
\text { productos para la extracción de aceite } \\
\text { rojo y palmiste, procesamiento de } \\
\text { raquis, plantas de compost. } \\
\text { Productos para la minería: ejecución } \\
\text { de obras, plantas para la producción de } \\
\text { explosivos. } \\
\text { Estructura: productos para el } \\
\text { transporte masivo, puentes peatonales, } \\
\text { edificaciones, tanques metálicos. } \\
\text { Biodiesel: maquinaria para la refinación } \\
\text { de aceites, biodiésel palma y destilado, } \\
\text { y operación y mantenimiento. }\end{array}$ & $\begin{array}{l}\text { Empresa de ingeniería y manufactura } \\
\text { metalmecánica, especializada en brindar } \\
\text { soluciones integrales para la industria } \\
\text { en general, a través de actividades de } \\
\text { diseño, fabricación, reparación, montajes } \\
\text { y asesoría técnica de proyectos. }\end{array}$ \\
\hline $\begin{array}{l}\text { INDUSTRIAS LAVCO } \\
\text { LTDA }\end{array}$ & $\begin{array}{l}\text { Fundición: hierro gris, nodular, bronce, } \\
\text { aluminio } \\
\text { Automotriz: camisas secas y húmedas } \\
\text { para motores a gasolina, camisas para } \\
\text { motores Diesel (húmedas y ceja), bujes } \\
\text { para asientos de válvulas } \\
\text { Industrial: fabricación de camisas } \\
\text { para grandes motores, partes para } \\
\text { compresores, ensamble y mecanizado } \\
\text { de piezas industriales, reparaciones } \\
\text { de cilindros motrices y compresores } \\
\text { reciprocantes reparación de piezas } \\
\text { industriales, desarrollo de soluciones, } \\
\text { operación y mantenimiento. }\end{array}$ & $\begin{array}{l}\text { Producción y comercialización de camisas } \\
\text { en fundición de hierro gris centrifugado } \\
\text { para la gran mayoría de automotores } \\
\text { diesel y gasolina, motores estacionarios } \\
\text { industriales, compresores y bombas } \\
\text { reciprocantes en gran variedad de rangos; } \\
\text { prestación del servicio de reparación de } \\
\text { cilindros compresores reciprocantes, } \\
\text { fabricación de pistones en hierro gris y } \\
\text { aluminio, y reparación de partes para } \\
\text { equipos del sector industrial. }\end{array}$ \\
\hline $\begin{array}{l}\text { INDUSTRIAS } \\
\text { TANUZI S.A. }\end{array}$ & $\begin{array}{l}\text { Desarrollo de productos: sistema } \\
\text { calador y elevación envasadora HK, } \\
\text { sellador horizontal de bolsas, grupos de } \\
\text { manejo, igualador de tensiones, sistema } \\
\text { rotativo de llantas, reductor planetario, } \\
\text { pistola neumática aturdidor. } \\
\text { Fabricación de maquinaria: enjuagadora } \\
\text { de botellas, tolva dosificadora de tapas, } \\
\text { transportador neumático y magnético } \\
\text { de tapas, paletizadora, cerradora } \\
\text { cierres, zaranda vibradora para pallets. } \\
\text { Fabricación de repuestos: troquel } \\
\text { formpack, bloque de bobinas, gato de } \\
\text { pechera, ejes, catalinas y carter para } \\
\text { locomotora U-18 Y U-20, pinza de } \\
\text { agarre para botella PET. } \\
\text { Mantenimiento: reparación de } \\
\text { transmisiones, sistema elevación } \\
\text { paletizadora, línea de envase de licores } \\
\text { y reparación de zapatas, sprokets y } \\
\text { bombas. }\end{array}$ & $\begin{array}{l}\text { Empresa dedicada al diseño, adaptación y } \\
\text { fabricación de repuestos, mantenimiento, } \\
\text { actualización y reconstrucción general de } \\
\text { maquinaria industrial. }\end{array}$ \\
\hline
\end{tabular}




\begin{tabular}{|c|c|c|}
\hline $\begin{array}{l}\text { TRANSEJES } \\
\text { COLOMBIA }\end{array}$ & $\begin{array}{l}\text { Cardanes } \\
\text { Ejes diferenciales } \\
\text { Embargues } \\
\text { Homocinéticos } \\
\text { Suspensión } \\
\text { Fuera de carretera }\end{array}$ & $\begin{array}{l}\text { Fabrica y distribuye productos y sistemas } \\
\text { tanto para fabricantes de equipos } \\
\text { originales (OEM) como para el mercado } \\
\text { de repuestos de automóviles e industrial. } \\
\text { Ofrece piezas de repuesto de calidad para } \\
\text { ejes cardanes, ejes diferenciales y juntas } \\
\text { homocinéticas. }\end{array}$ \\
\hline $\begin{array}{l}\text { METALÚRGICA DE } \\
\text { SANTANDER LTDA }\end{array}$ & $\begin{array}{l}\text { Servicios: soportes e ingeniería, } \\
\text { montajes, análisis de laboratorio y } \\
\text { ensayos no destructivos, reparación } \\
\text { de equipos y componentes, } \\
\text { mantenimientos de plantas industriales. } \\
\text { Fabricación: intercambiadores de calor, } \\
\text { molinos, tolvas y silos, mezcladores y } \\
\text { repuestos para todos los anteriores. } \\
\text { Fundición } \\
\text { Segmentos }\end{array}$ & $\begin{array}{l}\text { Compañía metalúrgica-metalmecánica } \\
\text { dedicada al desarrollo de productos y } \\
\text { servicios para satisfacer las necesidades } \\
\text { específicas de diferentes sectores } \\
\text { industriales. Realiza procesos de } \\
\text { fundición, taller de mecanizados con } \\
\text { CNC, taller de metalistería, departamento } \\
\text { de ingeniería y departamento de proyectos } \\
\text { especiales. }\end{array}$ \\
\hline FORJADOS S.A. & $\begin{array}{l}\text { Autopartes: dirección y suspensión, } \\
\text { cubos de rueda y embarque, transmisión, } \\
\text { estampados y troquelados. } \\
\text { Maquinaria agrícola } \\
\text { Componentes de alta tensión } \\
\text { Líneas férreas } \\
\text { Forja en bronce } \\
\text { Motopartes }\end{array}$ & $\begin{array}{l}\text { Fabricantes de piezas forjadas para la } \\
\text { industria en general mediante la forja por } \\
\text { estampa; productores de piezas en lámina } \\
\text { de acero de diversos espesores mediante el } \\
\text { proceso de troquelado y estampado. }\end{array}$ \\
\hline $\begin{array}{c}\text { PENAGOS HERMANOS } \\
\text { Y CÍA LTDA }\end{array}$ & $\begin{array}{l}\text { Línea Café: despulpadoras, secadoras } \\
\text { y lavadoras de café, equipos de } \\
\text { fermentación y molinos. } \\
\text { Línea agrícola: picapastos, ensiladora } \\
\text { manual, cosechadora de forrajes, } \\
\text { desgranadoras de maíz, trituradores, } \\
\text { picadores y molinos, picadora de hoja } \\
\text { de palma, trapiches horizontales y carro } \\
\text { mezclador (mixer). }\end{array}$ & $\begin{array}{l}\text { Empresa dirigida a proveer soluciones } \\
\text { para el sector de la agroindustria con } \\
\text { la fabricación de trapiches, motores } \\
\text { hidráulicos Pelton, tornos, taladros, entre } \\
\text { otros. Y fabricación de equipos para el } \\
\text { procesamiento del café. }\end{array}$ \\
\hline
\end{tabular}

Fuente: Confecamaras. 2016. Elaboración propia.

\section{Exportaciones del sector metalmecánico de Colombia}

Las exportaciones de la cadena del sector metalmecánico de Colombia son impulsadas principalmente por los departamentos de Antioquia, Bolívar y Cundinamarca representando conjuntamente el $75 \%$ de las exportaciones totales del sector en Colombia tal y como se refleja en la tabla 12 . Se destaca principalmente que el departamento de Bolívar concentra el $28,69 \%$ de las exportaciones de la cadena del sector metalmecánico de Colombia, seguido en segundo lugar por el departamento de Cundinamarca con el $27 \%$ y el departamento de Antioquia en tercer lugar con un $18.26 \%$ de participación de las ventas internacionales de la cadena. El departamento de Santander se encuentra en el séptimo lugar de ventas internacionales de la cadena 
metalmecánica aportando tan solo el $1,79 \%$ de las exportaciones de la cadena del país.

Tabla 12. Principales regiones exportadoras

\begin{tabular}{lc}
\hline \multicolumn{1}{c}{ Departamento } & \% exportaciones \\
\hline Antioquia & 18,26 \\
\hline Atlántico & 4,75 \\
\hline Bolívar & 28,69 \\
\hline Norte de Santander & 0,27 \\
\hline Caldas & 11,30 \\
\hline Cauca & 0,82 \\
\hline Cundinamarca & $27 \%$ \\
\hline Risaralda & 0,29 \\
\hline Santander & 1,79 \\
\hline Valle & 6,57 \\
\hline
\end{tabular}

Fuente: Ministerio de Comercio Industria y Turismo (2016). Elaboración propia.

En la tabla 13 se señala la baja participación de las exportaciones de Santander en valor de dólares americanos en la cadena del sector metalmecánico. En este cuadro se destaca el considerable decrecimiento en las ventas internacionales del departamento de Santander al pasar de tener una participación de un 5.5\% en las exportaciones de la cadena de Colombia en el 2010 con ventas por US\$21.943.925 a pasar a exportar tan solo US\$6.824.074 en el 2015 teniendo una caída porcentual en las ventas internacionales de $-3.7 \%$ en el período $2010-2015$.

Tabla 13. Participación de las exportaciones de Santander (dólares americanos)

\begin{tabular}{lccc}
\hline \multicolumn{1}{c}{ Departamentos } & $\mathbf{2 0 1 0}$ & $\mathbf{2 0 1 5}$ & CAP \% \\
\hline Santander & 21.943 .925 & 6.824 .074 & $-20,8 \%$ \\
\hline Part. \% & $5,5 \%$ & $1,8 \%$ & $-3.7 \%$ \\
\hline Total nacional & $\mathbf{3 0 0 . 0 6 2 . 1 2 2}$ & $\mathbf{3 8 1 . 6 5 4 . 1 3 4}$ & $\mathbf{0 , 9 \%}$ \\
\hline
\end{tabular}

Fuente: SIEX- DIAN (2016). Elaboración propia.

Se refleja que la participación de las exportaciones de Santander en volumen nacional es mínima, representando tan solo el $1,1 \%$ de las ventas internacionales de la cadena metalmecánica al aportar tan solo $872.834 \mathrm{~kg}$ en el 2015, presentando una dramática caída luego de tener una participación del 2,6\% en el 2010 con ventas por encima de $2.500 .000 \mathrm{~kg}$. 
Tabla 14. Participación de las exportaciones de Santander en volumen ( $k n$ \%)

\begin{tabular}{lccc}
\hline Departamentos & $\mathbf{2 0 1 0}$ & $\mathbf{2 0 1 5}$ & CAP \% \\
\hline Santander & 2.518 .393 & 872.834 & $-19,1 \%$ \\
\hline Part. \% & $2,6 \%$ & $1,1 \%$ & \\
\hline Total nacional & $\mathbf{9 8 . 5 3 8 . 9 5 0}$ & $\mathbf{7 9 . 3 3 0 . 3 8 7}$ & $\mathbf{- 4 , 2 \%}$ \\
\hline
\end{tabular}

Fuente: SIEX- DIAN (2016). Elaboración propia.

El comportamiento de los capítulos arancelarios correspondientes a la descripción de producto de herramientas y útiles, artículos de cuchillería y cubiertos de mesa de metal común; partes de estas manufacturas diversas de metal común; reactores nucleares, calderas, máquinas, aparatos y artefactos mecánicos; partes de estas máquinas o aparatos; máquinas, aparatos y material eléctrico y sus partes; aparatos de grabación o reproducción de sonido, aparatos de grabación o reproducción de imagen y sonido en televisión y las partes y accesorios de estos aparatos.

Tabla 15. Exportaciones capítulos 82-85

\begin{tabular}{rrrrrr}
\hline Cap./Año & \multicolumn{1}{l}{ 2011 } & \multicolumn{1}{c}{$\mathbf{2 0 1 2}$} & \multicolumn{1}{c}{$\mathbf{2 0 1 3}$} & \multicolumn{1}{c}{$\mathbf{2 0 1 4}$} & \multicolumn{1}{c}{$\mathbf{2 0 1 5}$} \\
\hline 82 & 9.933 & $31,516.49$ & $10,429.21$ & $13,011.29$ & $10,941.75$ \\
\hline 83 & $881,666$. & $681,739.76$ & $434,293.31$ & $490,204.89$ & $442,249.08$ \\
\hline 84 & $8,336,800$. & $11,153,916.63$ & $11,718,251.13$ & $10,624,843.36$ & $12,870,313.44$ \\
\hline 85 & $1,161,526$. & $1,089,919.98$ & $639,362.87$ & $1,802,494.65$ & $1,552,912.97$ \\
\hline
\end{tabular}

Fuente: SIEX- DIAN (2016). Elaboración propia.

El capítulo arancelario con mayores ventas internacionales es el 84 correspondiente a reactores nucleares, calderas, máquinas, aparatos y artefactos mecánicos; partes de estas máquinas o aparatos, teniendo exportaciones cuyo valor oscila entre los \$US 8 millones y los US\$ 12 millones en los años 2011-2015. El segundo capítulo arancelario de gran dinámica exportadora en la cadena metalmecánica del departamento de Santander es el 85 correspondiente a máquinas, aparatos y material eléctrico y sus partes; aparatos de grabación o reproducción de sonido, aparatos de grabación o reproducción de imagen y sonido en televisión y las partes y accesorios de estos aparatos con ventas superiores al millón de dólares en el período de análisis y se destaca el comportamiento exportador en el 2014 con ventas internacionales por US1.802.494.

Los destinos de las exportaciones del departamento de Santander de productos de la cadena metalmecánica estuvieron dirigidos en el período 2010-2015 principalmente a los mercados de los países vecinos. Ecuador ha sido el principal destino de las 
exportaciones de la cadena del sector metalmecánico del departamento de Santander con un $44.3 \%$ de las exportaciones del departamento. Venezuela fue un destino importante de las exportaciones de Santander entre los años 2010 y 2012, pero siendo un mercado nulo entre 2013 y 2015. Sin embargo, el aporte del mercado venezolano es importante en el período de análisis con una participación del $16.7 \%$ de las ventas internacionales del departamento. De igual forma es de resaltar la importancia adquirida en los dos últimos años por los socios de Colombia en la Alianza del Pacífico como son Chile a donde se dirigen el 5.6\% de las exportaciones del departamento de Santander y Perú con el 3.8\% convirtiéndose estos como los mercados con mejores perspectivas para las exportaciones de la cadena metalmecánica de Santander.

Es importante señalar que con la entrada en vigencia del Tratado de Libre Comercio con Estado Unidos este mercado se convierte en el tercer destino de las exportaciones de la cadena del sector metalmecánico del departamento, con un 14,5\%. La tendencia de las exportaciones del departamento de Santander se diferencia de lo sucedido en el resto del país donde el principal mercado de exportación es el de Estados Unidos, representando el $36,6 \%$ de las exportaciones totales de la cadena metalmecánica.

Tabla 16. Destinos de las exportaciones (valor en dólares americanos)

\begin{tabular}{|c|c|c|c|c|}
\hline \multirow{2}{*}{$\begin{array}{l}\text { Metalmecánica } \\
\text { Destinos }\end{array}$} & \multicolumn{2}{|c|}{ Valor } & \multicolumn{2}{|c|}{ Part \% en el total } \\
\hline & Colombia & Santander & Colombia & Santander \\
\hline Ecuador & 52.306 .476 & 3.025 .662 & $14,0 \%$ & $44,3 \%$ \\
\hline Venezuela & 35.678 .728 & 1.141 .577 & $9,5 \%$ & $16,7 \%$ \\
\hline Estados Unidos & 137.299 .480 & 991.748 & $36,6 \%$ & $14,5 \%$ \\
\hline Chile & 8.696 .826 & 385.192 & $2,3 \%$ & $5,6 \%$ \\
\hline Brasil & 6.312 .808 & 262.531 & $1,7 \%$ & $3,8 \%$ \\
\hline Perú & 25.153 .622 & 256.812 & $6,7 \%$ & $3,8 \%$ \\
\hline República Dominicana & 6.138 .017 & 246.628 & $1,6 \%$ & $3,6 \%$ \\
\hline Guatemala & 7.756 .263 & 140.695 & $2,1 \%$ & $2,1 \%$ \\
\hline Costa Rica & 7.843 .186 & 107.577 & $2,1 \%$ & $1,6 \%$ \\
\hline Uruguay & 715.059 & 94.559 & $0,2 \%$ & $1,4 \%$ \\
\hline Panamá & 23.900 .393 & 50.561 & $6,4 \%$ & $0,7 \%$ \\
\hline Bolivia & 4.597 .186 & 38.785 & $1,2 \%$ & $0,6 \%$ \\
\hline El Salvador & 3.974 .189 & 33.142 & $1,1 \%$ & $0,5 \%$ \\
\hline Puerto Rico & 1.587 .718 & 16.305 & $0,4 \%$ & $0,2 \%$ \\
\hline Honduras & 6.728 .108 & 15.965 & $1,8 \%$ & $0,2 \%$ \\
\hline Total Exportado & 374.830.059 & 6.824.074 & $100,0 \%$ & $100,0 \%$ \\
\hline
\end{tabular}

Fuente: SIEX- DIAN (2016). Elaboración propia. 
Según el Programa de Transformación Productiva (2016), en Colombia se identificaron en el 2015 un total de 1516 empresas exportadoras, de las cuales 811 se encuentran en el departamento de Cundinamarca representando el 53,4\% de las empresas a nivel nacional, seguido por el departamento de Antioquia con 302 , representando el $20 \%$ del total de empresas de la cadena metalmecánica. El departamento de Santander cuenta con 23 empresas exportadoras aportando tan solo el $1,5 \%$ del total nacional.

Tabla 17. Empresas exportadoras por departamento

\begin{tabular}{lc}
\hline \multicolumn{1}{c}{ Departamento } & $\begin{array}{c}\text { Número de empresas } \\
\text { exportadoras }\end{array}$ \\
\hline Antioquia & 302 \\
\hline Atlántico & 88 \\
\hline Bolívar & 50 \\
\hline Magdalena & 3 \\
\hline Córdoba & 1 \\
\hline Norte de Santander & 31 \\
\hline Cundinamarca & 811 \\
\hline Quindío & 4 \\
\hline Caldas & 18 \\
\hline Risaralda & 16 \\
\hline Santander & 23 \\
\hline Valle & 152 \\
\hline Tolima & 1 \\
\hline Cauca & 10 \\
\hline Nariño & 1516 \\
\hline Arauca & 5 \\
\hline Total & 150 \\
\hline & 16 \\
\hline
\end{tabular}

Fuente: DANE (2016). Elaboración propia.

\section{Conclusiones}

Según el Ministerio de Comercio, Industria y Turismo, el departamento de Santander cuenta con seis cadenas productivas con potencial exportador. Estas cadenas son: químicos, metalmecánico, industrias 4.0, moda, agroalimentario y turismo. 
En el marco de las Jornadas de Transformación productiva de Santander se tomó la decisión de apoyar el subsector de productos elaborados de metal. Los capítulos arancelarios con potencial exportador del departamento de Santander de la cadena priorizada del sector metalmecánico son los comprendidos entre los capítulos arancelarios 82 y 85 .

En generación de empleo en el ámbito nacional en el 2015 el sector metalmecánico aportó 203.386 puestos de trabajo en la economía colombiana, de los cuales 9502 se generaron en Santander y 6066 en el Área Metropolitana de Bucaramanga. El departamento de Santander se ubica en el quinto puesto respecto a los demás departamentos concentrando el 4,7\% del empleo de la cadena.

En producción industrial el departamento de Santander se ubica en la quinta posición con $4,1 \%$ de la producción de la cadena metalmecánica del país, concentrando 130 empresas equivalentes al 3,2\% de las empresas del total de empresas metalmecánicas del país. La ciudad de Bucaramanga es la que se destaca con un mayor número de empresas de la cadena del sector metalmecánico con 68 , correspondientes al 52\% del total de empresas del departamento.

En el indicador de participación de las exportaciones, el departamento de Santander aporta el 1,79\% de las exportaciones de la cadena. Sin embargo, se destaca un decrecimiento en las ventas internacionales del departamento de un $20.8 \%$ en el período 2010-2015 al pasar de exportar US\$21.943.925 en el 2010 a tan solo US\$ 6.824.074 en el 2015, teniendo una caída porcentual de -3.7\% en el período 2010-2015. Por su parte, el departamento de Santander cuenta con 23 empresas exportadoras aportando tan solo el 1,5\% del total nacional. El destino de las exportaciones del departamento de Santander de productos de la cadena metalmecánica estuvo dirigido en el período 2010-2015 principalmente a los mercados de los países vecinos: Ecuador con un $44.3 \%$ y Venezuela con una participación del $16.7 \%$ de las ventas internacionales del departamento, seguido muy de cerca por el mercado norteamericano con un $14,5 \%$.

\section{Referencias}

Barrientos, P. (2011). El clúster en la estrategia de marketing internacional. Semestre Económico, 14, 49-66.

Bagnasco, A. (2000). Nacimiento y transformación de los distritos industriales: Un examen de la investigación en Italia con observaciones de método para la teoría del desarrollo. En Desarrollo social y cambios productivos en el mundo rural europeo contemporáneo (pp. 59-91). Fondo de Cultura Económica.

Cámara de Comercio de Bucaramanga. (2016). Observatorio de Competitividad. Compite 360. 
Castellanos, O., Rojas, J., Villarraga, L. y Ustate, E. (2001, julio-diciembre). Conceptualización y papel de la cadena productiva en un entorno de competitividad. INNOVAR, Revista de Ciencias Administrativas y Sociales, 18, 87-98.

Confecamaras. (2016). Boletín. Lo que pasa en la Red de Cámaras de Comercio. http://www.confecamaras.org.co/boletines/460-red-julio-19-de-2016

Corrales, S. (2007). Importancia del clúster en el desarrollo regional actual. Frontera Norte, 19(37), 173-201.

Dalle, D., Fossati, V., \& Lavopa, F. (2013). Política industrial: ¿El eslabón perdido en el debate de las cadenas globales de valor? Revista Argentina de Economía Internacional, 2, 3-16.

DATLAS. (2016). Atlas Colombiano de Complejidad Económica. http:// datlascolombia.com/

Dirección de Impuestos y Aduanas Nacionales. DIAN. (2016). Sistema Integrado de Estadística de Comercio Exterior.

Humphrey, J., \& Schmitz, H. (2002). Las empresas de los países en vías de desarrollo en la economía mundial: poder y mejora de las cadenas globales de valor. Argentina: Instituto Nacional de Tecnología Industrial.

Isaza, J. G. (2008). Cadenas productivas. Enfoques y precisiones conceptuales. Sotavento MBA, 11, 8-25.

ITC. Trade Map. (2016). Estadísticas del comercio para el desarrollo internacional de las empresas Datos comerciales mensuales, trimestrales y anuales.

Kosakoff, B., \& López, A. (2008). América Latina y las cadenas globales de valor: debilidades y potencialidades. Globalización, competitividad y gobernabilidad de Georgetown. Universia, 2(1).

Lazzeretti, L. (2006). Distritos industriales,»clúster» y otros: un análisis «trespassing» entre la economía industrial y la gestión estratégica. Economía Industrial, (359), $59-72$.

Lima, S. M., Castro, A. M., Mengo, O., Medina, M., Maestrey, M., Trujillo, V. y Alfaro, O. (2001). La dimensión de entorno en la construcción de la sostenibilidad institucional.

López, C. (2003). Redes empresariales: Experiencias en la región Andina. Perú: Editorial Minka, Cooperación Italiana y CEPAL

ONUDI. (2004). Manual de mini cadenas productivas. Organización de las Naciones Unidas para el Desarrollo Industrial, República de Colombia: Ministerio de Comercio, Industria y Turismo, Bogotá. 
Ministerio de Comercio, Industria y Turismo de Colombia. (2016). Principales Regiones Exportadoras.

McCormick, D. (2005, enero-junio). El futuro de los clusters y las cadenas productivas. Semestre Económico, 87-102.

Programa de Transformación Productiva, Cadenas Priorizadas. Ministerio de Comercio, Industria y Turismo de Colombia. 2016.

Porter, M. (1990). La ventaja competitiva de las naciones. Harvard Business Review, 85(11), 69-95.

Richardson, G. B. (1972). The Organisation of Industry. The Economic Journal, 82(327), 883-896.

Solvell, O. (2008). Clusters balancing evolutionary and constructive forces (1a ed.). Stockholm, Sweden: Ivory Tower Publishing

Stumpo, G. (1996). Encadenamientos, articulaciones y procesos de desarrollo industrial.

Tomta, D., \& Chiatchoua, C. (2009). Cadenas productivas y productividad de las Mipymes. Criterio Libre, (11), 145-164. 